\title{
A behavior systems view of the organization of multiple responses during a partially or continuously reinforced interfood clock
}

\author{
KATHLEEN M. SILVA \\ University of Redlands, Redlands, California \\ and \\ WILLIAM TIMBERLAKE \\ Indiana University, Bloomington, Indiana
}

\begin{abstract}
We examined how a 50\% Pavlovian partial reinforcement (PRF) schedule, versus a 100\% continuous reinforcement $(\mathrm{CRF})$ schedule, altered the asymptotic amount and distribution of focal and general search behavior in rats during 48-sec trials with and without a four-segment interfood clock (S1-S2-S3-S4-US). Under CRF, but not PRF, average asymptotic focal search (nosing in the feeder) increased across the last two clock segments (S3 and S4), and more for the clock group than the no-clock group. Locomotor general search peaked in the second clock segment (S2) for the CRF-clock and CRF-no-clock groups and in S3 for the PRF-clock groups. Furthermore, the ratio of general search to maximum focal search was higher for the PRF-clock group than for the CRF-clock group. This pattern of results supports the view that predictable reward presentations temporally organize search states and related responses between food presentations. The relative expression of these responses varies with the predictability and proximity of reward and is more sharply defined in the presence of a clock.
\end{abstract}

Rats show an organized and predictable average sequence of responses during repeated fixed temporal intervals bounded by presentations of food reward. For example, during a range of interfood intervals (IFIs; $30-180 \mathrm{sec}$ ), rats, on average, first sniff in and near the feeder (often drinking at longer IFIs if water is available), followed by restless activity and wheel running and then by a return to nosing in the feeder (Lucas, Timberlake, \& Gawley, 1988; Reid, Bacha, \& Moran, 1993). Pigeons also show consistent response sequences; they begin an interval with search around the hopper, followed by locomotion away and back again and then by behavior directed toward the feeder wall (Innis, Simmelhag-Grant, \& Staddon, 1983; Staddon, 1977; Staddon \& Simmelhag, 1971; Timberlake \& Lucas, 1985).

These results are consistent with a behavior systems view that predictable food entrains a series of motivational search states expressed in organized sequences of related responses (Domjan, 1994; Timberlake, 1983, 1994, 2001; Timberlake \& Lucas, 1989; Timberlake \& K. M. Silva, 1995). Drawing from naturalistic observations and laboratory experiments of rat foraging (e.g., Barnett,

Grants NSF IBN 9408366 and 9817175 supported this work. We thank Thomas Gross and Francisco Silva for their helpful comments and suggestions. Please address correspondence to K. M. Silva, Department of Psychology, University of Redlands, P.O. Box 3080, 1200 East Colton Avenue, Redlands, CA 92373-0999 (e-mail: kathleen_silva@redlands.edu) or to W. Timberlake, Department of Psychology, Indiana University, Bloomington, IN 47405 (e-mail: timberla@indiana.edu).
1975; Ewer, 1971; Steiniger, 1950; Telle, 1966; Timberlake \& Washburne, 1989), the behavior systems approach to fixed-time (FT) schedules in rats predicts that, immediately after food, the animal enters a postfood focal search mode, characterized by behavior directed toward the feeder area (Lawler \& Cohen, 1992; Lucas et al., 1988; F. J. Silva, Timberlake, \& Cevik, 1998; K. M. Silva \& Timberlake, 1998b; Timberlake \& F. J. Silva, 1994). Toward the middle of the IFIs, the rat enters a general search mode characterized by locomotion around the experimental chamber (K. M. Silva \& Timberlake, 1997, 1998b). As the time to food delivery approaches, the rat enters a focal search mode characterized by behavior directed again toward the feeder (Lucas et al., 1988; F. J. Silva \& Timberlake, 2000; F. J. Silva, Timberlake, \& Gont, 1998; F. J. Silva, Timberlake, \& Koehler, 1996; K. M. Silva \& Timberlake, 1997, 1998a, 1998b, 1999; see also Burns \& Domjan, 1996). Finally, when food is delivered, the animal enters a handling/consuming mode, involving chewing and ingesting the food, before the sequence begins again.

To gain more precise control of the focal search responses that precede reward, researchers have used interfood clocks in FT schedules (an interfood clock is a sequence of different stimuli typically dividing the IFI into equal segments; Dinsmoor, 1994; Dinsmoor, Dougan, Pfister, \& Thiels, 1992; Dinsmoor, Lee, \& Brown, 1986; Ferster \& Skinner, 1957; Matthews, Bordi, \& Depollo, 1990; Matthews \& Lerer, 1987; Palya, 1985; Palya \& Bowers, 2003; Palya \& Pevey, 1987). To further investi- 
gate the relation of different motivational modes to interfood clocks, K. M. Silva and Timberlake (1998b) examined the distribution of rats' responses across clock segments over a variety of IFI durations. The clock consisted of a sequence of four equal-duration lights, each flashing at a higher rate (S1-S2-S3-S4). As was expected, the rats showed an average sequence of responses beginning with rearing near the feeder after food (during S1), followed by locomotion away from the feeder (during S2 and a portion of S3) and then nosing in the feeder (during the last portion of S3 and all of S4).

The extent to which a clock stimulus controls a search mode and related responses should depend on the temporal and spatial proximity of that stimulus to food. A stimulus that reliably and immediately precedes food should best control a focal search mode and appropriate responses, whereas a stimulus presented more temporally and/or spatially distant from food should better control a general search mode and related responses. Finally, a stimulus immediately following food should control a postfood search mode (F. J. Silva, Timberlake, \& Cevik, 1998; F. J. Silva et al., 1996; K. M. Silva \& Timberlake, 1997, 1998a, 1998b, 1999; Timberlake, 1994, 2001; Timberlake \& Lucas, 1989).

The sequence of explicit cues in an interfood clock appears to more sharply differentiate the temporal distribution of modes and responses (K. M. Silva \& Timberlake, 1998b, 1999). K. M. Silva and Timberlake (1998b) showed that when a four-segment interfood clock fills the IFI, activity near the feeder (i.e., postfood search) peaks during S1 (the first clock segment after food presentations), locomotion away from the food site (i.e., general search) peaks during S2 (the second segment in the clock), and nosing in the feeder (i.e., focal search) begins to rise in S3 and then rises more steeply in S4 (the final segment of the clock before a food presentation). A similar sequence of behavior occurs in the absence of the clock, but the responses are not as clearly demarcated across the IFI (see also K. M. Silva \& Timberlake, 1998a). For example, a rise in nosing in the feeder still occurs during the last half of the IFI (i.e., S3 and S4), but the rise is not as marked, particularly in the last quarter of the IFI (i.e., S4). As we will see, the demarcation of the IFI by a clock is useful for clarifying the effects of partial reinforcement on IFI responding.

The primary purpose of the present study was to examine how the distribution of multiple responses is organized across a 48 -sec trial in two partial $(50 \%)$ reinforcement (PRF) groups versus two continuous (100\%) reinforcement (CRF) groups. One PRF and one CRF group received 48 -sec trials with a four-segment clock; the other PRF and CRF groups received 48-sec trials with no clock. We chose a 50\% PRF schedule because it has been shown in a number of experiments to affect several responses to a single conditioned stimulus (CS). For example, when a light CS distant from the feeder consistently predicted a food unconditioned stimulus (US), rats under a CRF schedule generally first approached the light (i.e., sign tracking) and then approached the feeder (i.e., goal tracking) during the CS (Davey \& Cleland, 1982; Davey, Oakley, \& Cleland, 1981). In contrast, a PRF schedule produced a longer period of sign tracking and less goal tracking (Davey \& Cleland, 1982; see also Boakes, 1977, 1979). Timberlake (2001) found related results in groups trained with different CSs under different reward probabilities. Relative to a CRF schedule, PRF enhanced attention to a ball-bearing CS (a stimulus promoting general search behavior) but decreased nosing in the feeder to a proximate light CS. In the context of behavior systems, PRF enhanced a general search response, and CRF enhanced a focal search response.

A behavior systems view assumes that the reduction in reliability of reward under PRF has an effect similar to lengthening the CS-US interval. That is, under a PRF schedule, a particular temporal point in the trial is, on average (combining reward and nonreward trials), more distant from the US than it is under a CRF schedule and, on the whole, less predictable than under a CRF schedule. This should change the amount and distribution of responding across the trial, as compared with a CRF schedule, by producing less focal search behavior toward the end of the trial and relatively more general search behavior in the middle of the trial (see also Pecoraro, Timberlake, \& Tinsley, 1999). We would expect these effects to be clearer with a clock, because it better defines the distance to the reward (see K. M. Silva \& Timberlake, 1998a, 1998b).

Traditional Pavlovian accounts also predict less nosing in the feeder just before potential food presentations under PRF schedules than under CRF schedules, because a CS under PRF should be a weaker predictor of reward and, therefore, support less responding than a CS under CRF does (Mackintosh, 1974; Pavlov, 1927; although see Gibbs, Latham, \& Gormezano, 1978). Response weakening during PRF can be extended to previous segments of a trial clock by invoking higher order conditioning based on the clock stimulus most proximate to food. However, traditional Pavlovian accounts make no allowance for PRF's increasing the relative amount of general search behaviors, such as locomotion, or changing their distribution across clock segments. It also fails to account for increased responding in the presence of a light or a rolling ball-bearing that is presented temporally distant from a food US (K. M. Silva \& Timberlake, 1997; Timberlake, 2001; Timberlake, Wahl, \& King, 1982). Instead, because stimuli more temporally distant from food are weaker conditioned reinforcers, a higher order conditioning account of PRF effects predicts a marked decrement in general search responses distant from food.

Konorski's (1967) model of Pavlovian conditioning is similar to the behavior systems view in positing multiple classes of conditioned responses (CRs): consummatory (discrete responses directed toward the site of the US) and preparatory (diffuse attentional responses directed toward the CS). Furthermore, as with the behavior systems 
view, Konorski argued that preparatory CRs are more likely to be expressed during PRF than during CRF. An important difference for present purposes is that Konorski used standard Pavlovian conditioning procedures with restrained subjects and made the general distinction that consummatory responses were discrete, whereas preparatory responses were diffuse. In contrast, the behavior systems view draws on an ethological analysis of ecological sequences of appetitive behavior (Timberlake \& K. M. Silva, 1995), which does not limit general search behaviors to diffuse responses directed at the location of a CS. Other differences will be addressed further in the Discussion section.

\section{METHOD}

\begin{abstract}
Animals
Thirty-two naive female Sprague Dawley albino rats, approximately 90 days old at the start of the experiment, were maintained at $85 \%$ of their free-feeding weights throughout the experiment. They were housed in individual home cages in a colony room regulated by a 12:12-h light:dark cycle and were fed approximately $12 \mathrm{~g}$ of commercial rat chow in their cages after each session. Water was always available in their home cages. They were divided into four groups of 8 rats each, matched according to weight.
\end{abstract}

\section{Apparatus}

The rats were tested in four identical chambers. Each chamber was a rectangular sheet metal box $60 \times 30 \times 30 \mathrm{~cm}$, with a Plexiglas top and front. The food receptacle was $2.0 \times 5.0 \times 1.8 \mathrm{~cm}$ deep and was located on a side wall $12 \mathrm{~cm}$ toward the back of the chamber. Food reward was delivered by a Waltke feeder (Waltke Scientific Enterprises, Psychology Department, Indiana University), which delivered two 45-mg Noyes pellets into the feeder. An infrared beam projected across the entrance of the feeder, an $11 \times$ $10 \mathrm{~cm}$ platform was located on the floor in front of the feeder, and a $9 \times 14 \mathrm{~cm}$ platform was located on the floor along the back of the chamber. The trial clock stimuli were presented on a green jewel light located on the feeder wall $5 \mathrm{~cm}$ from the back of the chamber and $6 \mathrm{~cm}$ above the chamber floor. A schematic of the apparatus is shown in K. M. Silva and Timberlake (1998a, 1998b). The experimental room also contained a video camera connected to a monitor in an adjacent room.

\section{Procedure}

Experimental sessions were conducted at the same time daily, 6 days per week. There were 20 trials. Each session terminated immediately after the 20th trial. The experimental procedure consisted of three phases: chamber adaptation, feeder training, and periodic food training.

Chamber adaptation. The rats were put in the chambers for $10 \mathrm{~min}$ on two successive days. No food or stimuli were presented. In their home cages, the rats were fed the same food pellets that they received during subsequent phases.

Feeder training. Five food pellets were placed in front of the feeder at the beginning of a session, and 20 pellets were placed in the feeder. A session terminated after the rats had eaten all the pellets or after $30 \mathrm{~min}$ had elapsed, whichever occurred first. A rat was considered trained when it ate 20 food pellets within a 30 -min session for two consecutive days.

Periodic food training (clock and no-clock groups). Four groups of rats (CRF-clock, PRF-clock, CRF-no-clock, and PRF-noclock) were exposed to 16 sessions consisting of 20 trials each. For the two clock groups (CRF-clock and PRF-clock), trials consisted of a sequence of four 12-sec lights flashing at different rates (i.e., the interfood clock). The first light (S1) was steady; the second light (S2) flashed on:off at a rate of $1 / \mathrm{sec}$; the third light (S3) flashed on:off at a rate of $2 / \mathrm{sec}$; and the fourth light (S4) flashed on:off at a rate of $10 / \mathrm{sec}$. Food pellets were presented immediately after the offset of the last stimulus (i.e., S1-S2-S3-S4-food) on $100 \%$ of the trials for the CRF-clock group and on $50 \%$ of the trials for the PRF-clock group. Although the order of the flashing rates was not counterbalanced in this study, previous research has shown that the pattern of responses during the clock is the same whether the clock stimuli flash progressively more quickly or more slowly or when the flashing rate is mixed (K. M. Silva, 1996). For the two no-clock groups (CRF-no-clock and PRF-no-clock), trials consisted of a 48-sec interval. The CRF-no-clock group received food every $48 \mathrm{sec}$ in the absence of a clock. The PRF-no-clock group received the same order of reinforcers as the PRF-clock group, but without a clock. During each trial for these no-clock groups, the light was lit but did not change across the trial, to avoid providing any external indicators of time. At the end of reinforced trials for all the groups, the light was darkened, and food was dispensed; at the end of nonreinforced trials for the PRF groups, the light was darkened for $4 \mathrm{sec}$, the amount of time it took for food to be dispensed on reinforced trials.

Our main concern was to control for number of trials, so all the groups received 320 trials, with the light darkened at the end of each trial (16 sessions with 20 trials per session). Because half of these 320 trials produced reinforcement for the PRF groups, we ran the PRF groups for an additional 320 trials (16 sessions) in order to equate the total number of reinforcers with that for the CRF groups. However, because the PRF groups showed no differences in responding between the end of the 16 sessions (average of last 4 days) and that of the 32 sessions (average of last 4 days) for the two dependent measures [nosing in the feeder and far-platform measures described below; $F \mathrm{~s}(1,7)<1.53, p>.05$ ], we will report only the data from the first 16 sessions for all the animals.

Dependent measures and data analysis. Two dependent measures were automatically recorded by computer-controlled devices: the durations of (1) nosing in the feeder, a measure of focal search, and (2) activating a platform located far from the feeder, a measure of general search. To be consistent with previous studies from our lab, we will refer to the latter measure as far-platform locomotion. Furthermore, the number of seconds spent nosing in the feeder and on the far platform during each trial segment was divided by $12 \mathrm{sec}$, the length of each trial segment. Hence, the nosing and far-platform data will be labeled as the proportion of time nosing in the feeder and proportion of time on the far platform, respectively, in the Results section and in the figures. All the rats reached stable levels of responding for both dependent measures by the 10th day of training. All statistically significant effects are reported at the $p<.05$ level.

\section{RESULTS}

Figure 1 shows asymptotic average proportion of time spent nosing in the feeder during each trial segment, and Figure 2 shows the asymptotic average proportion of time spent on the far platform during each trial segment. Each measure is averaged over the last four sessions for the CRF-clock, PRF-clock, CRF-no-clock, and PRF-noclock groups. Statistical tests consisted of a repeated measures analysis of variance (ANOVA) with which the influence of reinforcement type (CRF vs. PRF), presence of clock (clock vs. no clock), and trial segment (S1 vs. S2 vs. S3 vs. S4) on both dependent measures was examined. Fisher's least significant differences (LSD) tests were used to determine differences between trial segments. 


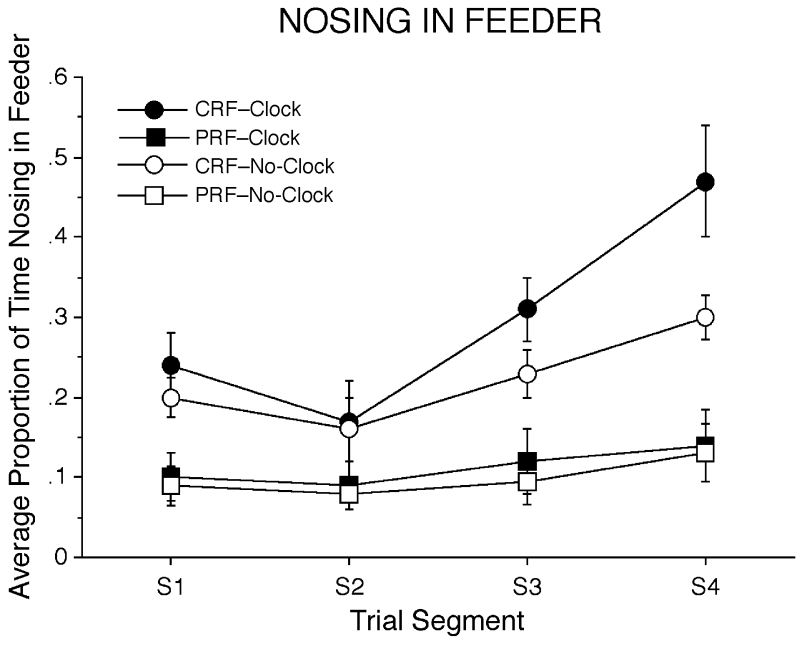

Figure 1. The average proportion of time spent nosing in the feeder during each trial segment (S1, S2, S3, and S4) averaged over the last four sessions of interfood clock training for the CRF-clock, PRF-clock, CRF-no-clock, and PRF-no-clock groups.

\section{Nosing in the Feeder Across the IFI at Asymptote}

CRF versus PRF. Figure 1 shows that nosing in the feeder, a typical focal search response, was generally higher for both CRF groups than for the PRF groups, a result confirmed by a main effect of reinforcement type $[F(1,28)=21.19]$. A significant interaction between reinforcement type, presence of clock, and trial segment $[F(3,84)=3.03]$ revealed a difference between the groups in levels and patterns of responding across the trial. In comparing the two clock groups, the average proportion of time spent nosing in the feeder significantly increased across the last half of the trial for the CRF-clock group, but not for the PRF-clock group. LSD tests revealed that nosing in the feeder decreased between $\mathrm{S} 1$ and $\mathrm{S} 2$ for the CRF-clock group, but increased between $\mathrm{S} 2$ and $\mathrm{S} 3$, and further increased between S3 and S4 during the last half of the trial. For the PRF-clock group, nosing did not show any significant increase across clock stimuli. For the two no-clock groups, nosing in the feeder increased across the last half of the trial (S3 and S4) for the CRF-noclock group, but not for the PRF-no-clock group. LSD tests revealed that nosing showed a gradual increase between S2 and S4 for the CRF-no-clock group. Furthermore, during all the trial segments, nosing was higher for the CRF groups (CRF-clock and CRF-no-clock) than for their PRF counterparts (PRF-clock and PRF-no-clock).

Clock versus no-clock. The extent to which the clock stimuli facilitated nosing in the feeder at asymptote was also examined by comparing the CRF-clock group with the CRF-no-clock group and the PRF-clock group with the PRF-no-clock group, averaged over the last 4 days. For the CRF groups, nosing in the feeder changed differently across trial segments for each group. An LSD test revealed that during the last half of a trial, nosing for the
$\mathrm{CRF}$-clock group increased between $\mathrm{S} 2$ and $\mathrm{S} 3$ and further increased between $\mathrm{S} 3$ and S4. For the CRF-no-clock group, nosing increased only between the second and the fourth quarters of a trial. Since the groups appeared to differ in their levels of nosing in the feeder toward the end of a trial, responding during the last two segments was compared, which confirmed that nosing was higher for the CRF-clock group than for the CRF-no-clock group. For both PRF groups, nosing in the feeder was low and did not change across trial segments.

In sum, the probability of reinforcement $(100 \%$ vs. $50 \%$ ) and the presence of an interfood clock (clock vs. no-clock) interacted to affect the asymptotic amount of nosing in the feeder (focal search) and its distribution across trial segments. When all the trials were followed by food (CRF), the clock stimuli facilitated nosing during the last half of the trial. Overall, PRF schedules produced less nosing than CRF schedules did, and there was no effect of the clock.
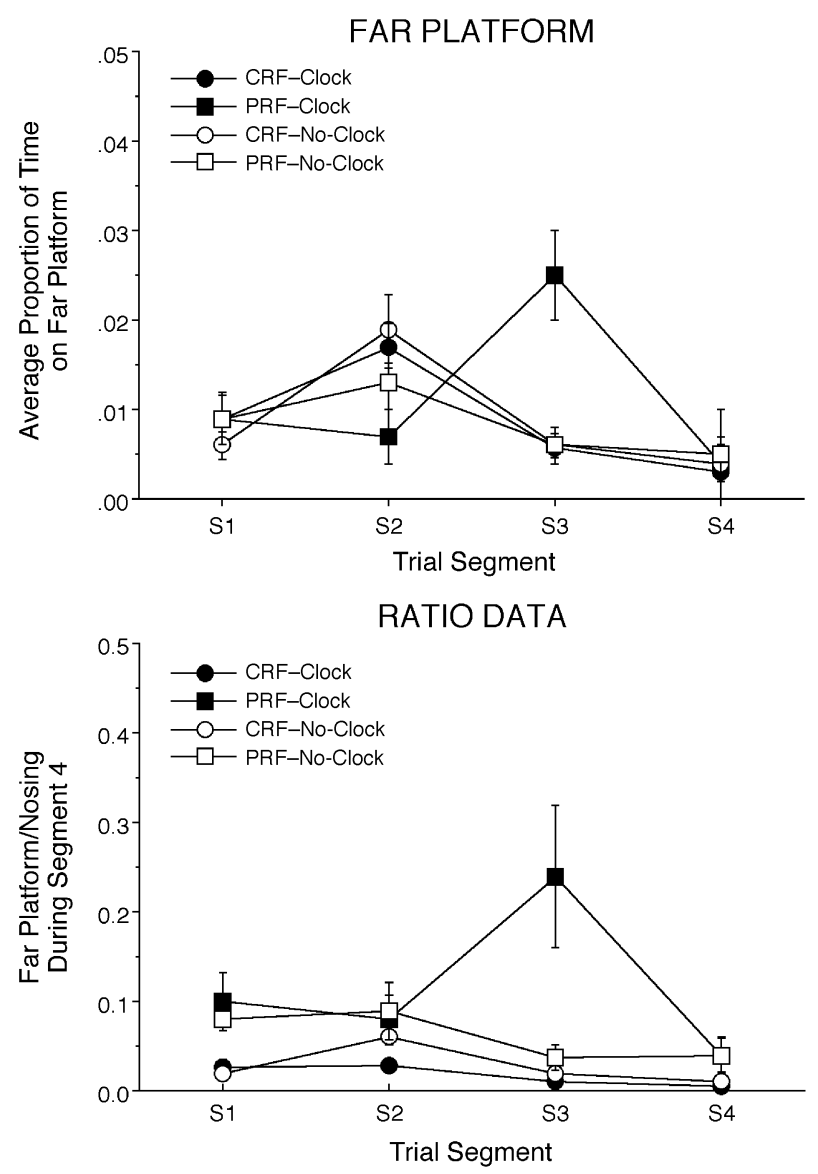

Figure 2. The top graph shows the average proportion of time spent on the far platform during each trial segment (S1, S2, S3, and S4) averaged over the last four sessions of interfood clock training for the CRF-clock, PRF-clock, CRF-no-clock, and PRF-no-clock groups. The bottom graph shows the ratio of farplatform locomotion to maximum focal search (in S4) averaged over the last four sessions of interfood clock training for the CRF-clock, PRF-clock, CRF-no-clock, and PRF-no-clock groups. 


\section{Locomotion on the Far Platform Across the IFI at Asymptote}

CRF versus PRF. The top graph of Figure 2 shows that the average proportion of time spent on the far platform at asymptote (a measure of general search averaged over the last 4 days of training) changed across trial segments for all the groups, showing a peak toward the middle of the trial, a result confirmed by a main effect of trial segment $[F(3,84)=10.57]$. A significant interaction between reinforcement type, presence of clock, and trial segment $[F(3,84)=3.67]$ revealed further differences in levels and patterns of far-platform locomotion across the trial segments among the groups. Comparing the two clock groups, LSD tests confirmed that far-platform locomotion peaked during different clock stimuli. The CRF-clock group's peak occurred during S2, whereas the PRF-clock group's peak occurred during S3. Furthermore, the PRF-clock group's peak was higher than the CRF-clock group's peak. The CRF-no-clock group also showed a peak in farplatform locomotion during S2, whereas the PRF-noclock group showed no systematic change across the trial.

Clock versus no-clock. The extent to which the clock stimuli facilitated movement on the far platform at asymptote, relative to when the clock was absent, was examined by comparing the CRF-clock group with its CRF-no-clock counterpart and the PRF-clock group with its PRF-no-clock counterpart. For the CRF groups, far-platform locomotion changed similarly across trials with and without the clock. LSD tests revealed that both groups spent more time on the far platform during the second quarter than during the first and fourth quarters of the trials, and there were no significant differences between the groups during any quarter of the trial. For the PRF groups, far-platform locomotion showed a distinct peak in the middle of the trial for the PRF-clock group, but little systematic change across the trial segments for the PRF-no-clock group. LSD tests revealed that the PRF-clock group spent more time on the far platform during S3 than during S1, S2, and S4 and that its peak during $\mathrm{S} 3$ was higher than the PRF-no-clock group's responding during all the trial segments.

In sum, when IFIs were reinforced $50 \%$ of the time (PRF groups), an interfood clock facilitated locomotion (a general search response) during the third quarter of the trial, as compared with the PRF-no-clock and the CRF groups. When all the trials ended in reinforcement (CRF), the presence of a clock had little effect on the pattern of locomotion across the trial. Locomotion peaked during the second quarter of the trial regardless of whether the clock was present or absent.

It is worth noting that the absolute proportion of time spent on the far platform was small (an average of 0.3 out of every $10 \mathrm{sec}$ in the highest clock segment). This was not surprising, because the measure recorded only time passing through a $3 \times 6$ in. area located along the back wall close to the end of the chamber away from the feeder. The key issue, though, is not the size of the measure, but its reliability and sensitivity, which, given that the measure distinguished among groups, was in an acceptable range. Further evidence that the far-platform measure is a reliable and sensitive measure of locomotion comes from previous studies that have produced results similar to those in the present study (K. M. Silva, 1996; K. M. Silva \& Timberlake, 1998b, 1999) and similar to those obtained when behavioral coding of videotape was used to measure locomotion around the chamber during interfood clocks (K. M. Silva, 1996).

\section{Ratio of Far-Platform Locomotion to Nosing in the Feeder During S4 at Asymptote}

A final way to examine the effects of PRF, relative to $\mathrm{CRF}$, on asymptotic responding is to determine the ratio of the amount of general search (far-platform locomotion) to the maximum amount of focal search (nosing in the feeder during S4). Both Boakes (1977) and Davey and Cleland (1982) have suggested that PRF should shift the relative amounts of nosing-in-the-feeder and awayfrom-the-feeder responses. The behavior systems approach (Timberlake \& Lucas, 1989) argues that although PRF should decrease the amount of focal search, it should produce relatively more general search. Importantly, the prediction of more general search under PRF than under $\mathrm{CRF}$ is relative rather than absolute. A prediction couched in absolute terms claims that the leanest PRF schedule should produce the greatest amount of general search. However, there is a limit as to how lean a reinforcement schedule can be used and still maintain responding. Given a minimal rate of food (e.g., 10\% of trials) and no opportunity to leave, rats are likely to become inactive (Timberlake \& K. M. Silva, 1995; see also F. J. Silva, K. M. Silva, \& Pear, 1992, for a similar effect with pigeons).

The bottom graph of Figure 2 shows the ratio of farplatform locomotion to maximum nosing in the feeder (in S4) during each trial segment, averaged over the last four sessions for all the groups. The higher the ratio, the greater the amount of locomotion, relative to nosing in the feeder. An ANOVA showed a significant interaction between CRF and PRF schedules and the presence versus the absence of the clock $[F(3,84)=5.37]$. Comparing only the CRF and the PRF clock groups, it can be seen that the PRF-clock group showed a higher ratio of general search to focal search than did the CRF-clock group $[F(1,28)=11.26]$. LSD tests confirmed the significance of this difference for the first three clock segments (S1, S2, and S3), with the largest significant difference during S3 ( $p=.000001)$. The no-clock groups showed no differences overall or during any trial segment. In short, the PRF-clock group showed a higher ratio of the index of general search, relative to maximum focal search, than did the CRF-clock group (and both the no-clock groups).

It is worth noting that, as compared with the top graph of Figure 2, the only peak of relative locomotion across clock segments in the bottom graph of Figure 2 occurs in S3 for the PRF-clock group. The peaks in far-platform locomotion during segment $\mathrm{S} 2$ shown by the two CRF 
groups in the top graph do not reappear reliably in the ratio of general search to focal search of the bottom graph. This indicates that the individual levels of general search creating the peaks during S2 in the CRF groups were accompanied by proportional levels of focal search. Only the PRF groups showed a tendency toward proportionally greater general search.

\section{Changes in Search Behavior Across Trial Blocks}

Figure 3 shows how responding changed across 2-day blocks for the four trial segments (S1, S2, S3, and S4) for each group (CRF-clock, PRF-clock, CRF-no-clock, and PRF-no-clock). The left column shows the proportion of time spent nosing in the feeder, and the right column shows the proportion of time spent on the far platform. The top graph in each column shows segment S4, with clock segments S3, S2, and S1 arranged below. A repeated measures ANOVA was performed to examine how responding during each trial segment changed over the eight 2-day training blocks for each group, and LSD tests determined between which blocks differences occurred. In terms of nosing in the feeder (left column) during $\mathrm{S} 3$ and $\mathrm{S} 4$, the CRF-clock group showed the greatest increase across 2-day blocks $[F \mathrm{~s}(7,49)>10.07]$, with nosing higher during the last four blocks than during the first three blocks for both segments. The CRF-no-clock group showed a slight increase across blocks $[F \mathrm{~s}(7,49)>$ 2.29], with nosing higher during the last block than during the first three blocks. Neither of the PRF groups showed systematic changes across blocks during S3 or S4 $[F \mathrm{~s}(7,49)<1.79]$. During S1 and $\mathrm{S} 2$, none of the interval segments showed any systematic changes across 2-day blocks $\left[F_{\mathrm{s}}(7,49)<1.67\right]$.

As indexed by proportion of time on the far platform (rightmost column of graphs in Figure 3), locomotion changed across 2-day blocks in all the segments $[F \mathrm{~s}(7,49)>2.40]$, although the pattern of change differed considerably from that for nosing in the feeder. One difference was the trend for locomotion in both clock groups in all the segments to begin high in the first block and then decrease over blocks. An exception to this trend was shown by the PRF-clock group in S3 and by the CRF-clock group in S2. For S3, the segment in which the PRF-clock group showed a peak in locomotion at asymptote (see the top graph in Figure 2), Figure 3 shows that this peak was the result of locomotion on the far platform remaining high across training blocks. Similarly, for S2, the segment in which the CRF-clock group showed a peak in locomotion at asymptote (see the top graph in Figure 2), locomotion on the far platform also remained high and unchanged. In contrast, the peak in locomotion during S2 for the CRF-no-clock group was produced by an increase across blocks, with locomotion higher in Blocks 7 and 8 than in Blocks 1-4. Finally, there was a tendency in all the segments for the PRF-noclock group to show a small increase in locomotion over blocks, followed by a decrease in the last several blocks.
In short, with the exception of the PRF-no-clock group, the groups showed similar patterns of organization on one or both measures; nosing in the feeder increased during S3 and S4, whereas far-platform locomotion increased or was sustained during one of the middle segments. The peak locomotion during $\mathrm{S} 2$ for the $\mathrm{CRF}$-clock group and during S3 for the PRF-clock group actually emerged as a result of a decrease in locomotion in the other segments. Finally, the tendency for the clock groups to show more initial locomotion than the no-clock groups did indicates an initial salience of the clock in controlling locomotion. It is worth noting that although the PRF-noclock group showed no significant linear trends in our measures of focal search or general search, inspection of videotapes revealed that learning occurred as an increase in approach to the feeder area over blocks during the final trial segments; but this occurred in the absence of increased nosing in the feeder.

\section{DISCUSSION}

The present study showed that at asymptote, nosing in the feeder (a measure of focal search) increased across the last two segments of the interfood clock during CRF (when the clock always terminated with food) but that the same response was relatively unchanged across clock segments during PRF (when the clock ended with food $50 \%$ of the time). At the same time, activation of the far platform (a measure of general locomotor search) was highest during the middle segments of the trial - the second quarter (S2) of the trial under CRF, but the third quarter (S3) of the trial under PRF.

Further analyses revealed that the asymptotic peak of far-platform locomotion in S3 for the PRF-clock group was accompanied by a much higher ratio of general search to focal search (calculated by dividing the far-platform time by the time spent nosing in the feeder during S4) than the ratio for both the CRF-clock and the CRF-no-clock groups. That is, during a partially reinforced clock, the general search peak in S3 was substantially higher than its relative amount of nosing in the feeder during S4. These data, and the data showing that the PRF-clock group showed relatively more general than focal search during the first three clock segments, are compatible with work in which single-CS Pavlovian procedures were used that showed that PRF schedules produce more behavior away from the feeder than near the feeder (Boakes, 1977; Davey \& Cleland, 1982; F. J. Silva et al., 1992) and with the demonstration by Pecoraro et al. (1999) of increased general search of a radial maze, relative to focal search, when the amount of reward on a specific arm was reduced.

The present study also showed that clock stimuli sharpened the expression of general and focal search behaviors in clock, as opposed to no-clock, versions of PRF and CRF. For rats exposed to trials with and without an interfood clock during CRF (CRF-clock and CRF-noclock groups, respectively), the most salient effect was 

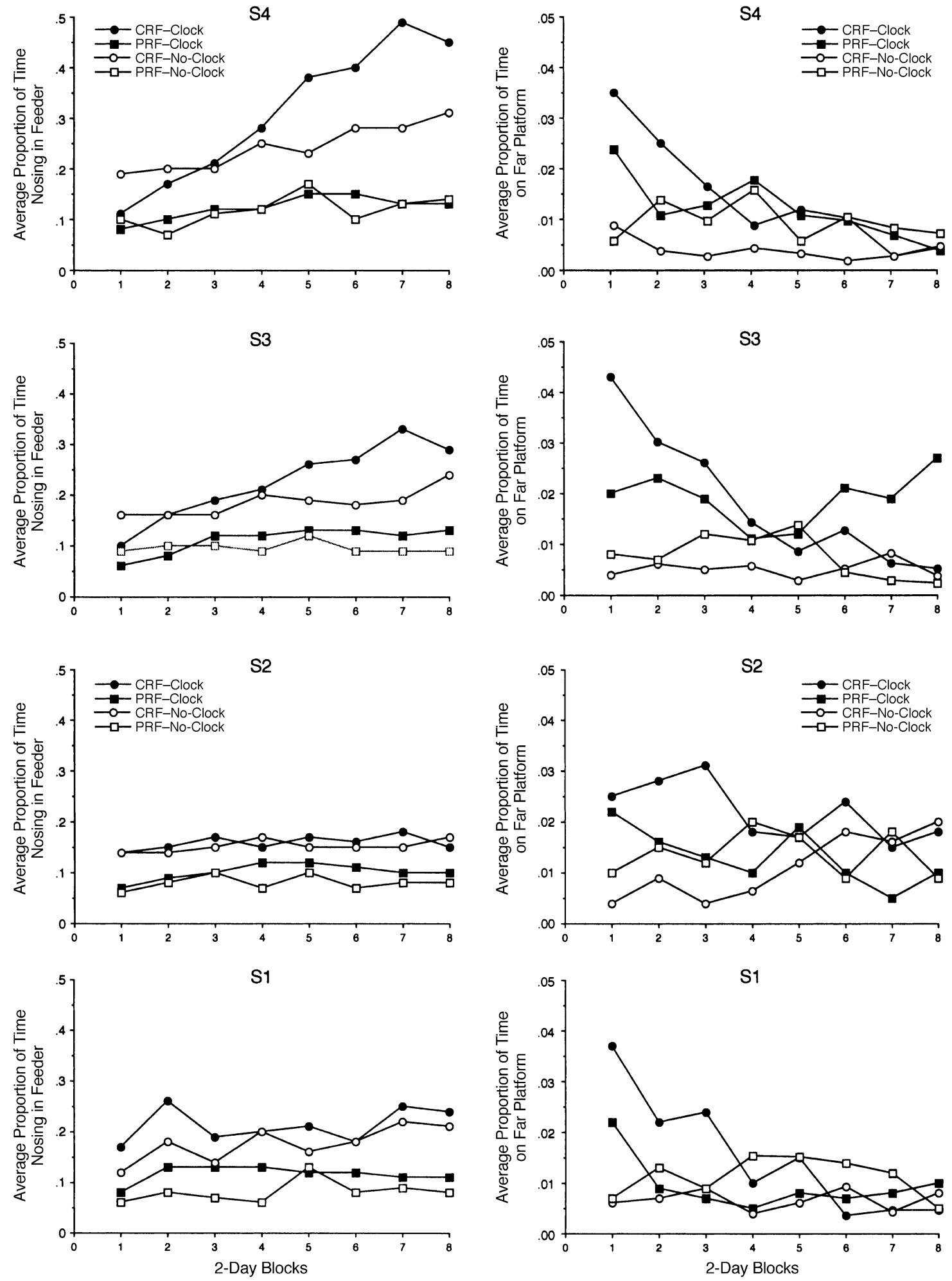

Figure 3. The average proportion of time spent nosing in the feeder (left column) and on the far platform (right column) averaged over 2-day blocks of training for the CRF-clock, PRF-clock, CRF-no-clock, and PRF-no-clock groups during each trial segment (S1, S2, S3, and S4). The segments are ordered starting with S4 in the top graph, and with the remaining segments displayed in descending order (S3, S2, and S1). 
observed in the CRF groups' focal search behavior. Both groups' nosing increased during the last half of the trial segments, but their absolute levels during the last two segments differed. The CRF-clock group nosed in the feeder more than the CRF-no-clock group did during the last two segments, a replication of a previous result (K. M. Silva \& Timberlake, 1998b). The 50\% clock and noclock conditions (PRF-clock and PRF-no-clock, respectively) showed little systematic change in nosing across trial segments.

In contrast, PRF differentially affected the pattern of general search behavior across trial segments for the PRF groups, but not for the CRF groups. For the rats exposed to trials with and without an interfood clock during CRF, far-platform locomotion peaked in the second quarter of the trial segments, also replicating a result previously obtained (K. M. Silva \& Timberlake, 1998b). For the rats exposed to trials with and without an interfood clock during PRF, locomotion peaked during S3 for the PRF-clock group but showed little systematic change across the trial segments for the PRF-no-clock group. In sum, during CRF, locomotion peaked during the second quarter of the IFI whether or not the clock was present. However, during PRF, locomotion peaked during the third quarter of the trial, but only in the presence of the clock.

Finally, the asymptotic patterns of general and focal search responses shown during the final two blocks of training (see Figure 1 and the top graph of Figure 2) came about differently over training blocks (see Figure 3). For focal search responses, nosing in the feeder emerged over the last half of the training blocks. In contrast, the higher level of far-platform locomotion for the $\mathrm{CRF}$-clock group in Segment 2 and the PRF-clock group in Segment 3 came about by maintaining the high level of locomotion shown in the first training blocks, whereas responding during the adjacent segments decreased over blocks.

\section{Traditional Pavlovian Accounts}

A fundamental difficulty with most Pavlovian explanations of the present data is the lack of a principled way to deal with the multiple responses that emerge or are maintained during different segments of the IFI (Fanselow, 1994; Holland, 1980; Palya, 1985; Timberlake \& K. M. Silva, 1995). As a result, such accounts do not clarify which sorts of responses are relevant to effects at different distances from a reward (K. M. Silva \& Timberlake, 1997; Timberlake, 1983, 1994). Higher order conditioning is one associative construct that is most easily applied to behavior during interfood clocks. In this view, the final stimulus segment (S4) acquires the most associative strength, which successively diffuses back to earlier stimuli, so that each previous segment has less associative strength than the one following it.

Higher order conditioning may explain the increase in nosing in the feeder across the IFI in the CRF group, but it cannot readily explain why the locomotion shown in this and previous studies and the ball-bearing contact shown in previous studies peak during $\mathrm{S} 2$ and $\mathrm{S} 3$. These responses occur in the middle portions of the trial and are scarcely present during S4 (K. M. Silva \& Timberlake, 1998a, 1998b, 1999). Furthermore, traditional Pavlovian accounts suggest that PRF should decrease conditioned responding (Mackintosh, 1974; Pavlov, 1927), a result not consistently obtained (cf. Gibbon \& Balsam, 1981; Gibbs et al., 1978; F. J. Silva et al., 1992). In fact, PRF supports responding that extends from food delivery back to the stimuli in the middle of the trial when an interfood clock is used and pigeons are the subjects (Palya \& Pevey, 1987).

Konorski's (1967) model has been used to make predictions about PRF effects (Boakes, 1977, 1979), and it resembles a behavior systems account in positing multiple (two rather than three) motivational classes of behavior, preparatory and consummatory. Behaviors in the preparatory class are characterized as more diffuse and unspecific, consisting of attentional responses directed toward the CS; behaviors in the consummatory class are characterized as definite and discrete responses directed toward the site of the US. Most important for our purposes here, Konorski argued that a preparatory $\mathrm{CR}$ is more likely to be expressed during PRF because the weakened CS is less able to elicit the representation of the US.

It is important to note that Konorski (1967) developed the preparatory-consummatory distinction in the context of more restricted Pavlovian procedures and that he differentiated between preparatory and consummatory behaviors primarily in terms of nervous-system-related physiology, rather than ethological form. As a result, he described preparatory behavior in terms of diffuse excitement, instead of the many specific discrete behaviors that can be observed in a less constrained circumstance. At best, his categories appear limited to subsets of the focal search and consummatory categories of behavior systems.

As a result, it is difficult to say what Konorski (1967) would have predicted about animals' behavior under less constrained conditions. However, there is evidence for discrete preparatory behaviors. For example, Jenkins, Barrera, Ireland, and Woodside (1978) showed that an unrestrained dog receiving food under CRF approached a light CS predicting food and proceeded to beg food from the light by barking, play-bowing, and nuzzling the light. Although directed at the CS, these were not diffuse search behaviors but, rather, specific search behaviors related to social food begging. We prefer to think that Konorski would have viewed behavior systems as a compatible framework for his own work, but we cannot say, nor does it seem appropriate to use his theory to make predictions about specific preparatory responses.

\section{Behavior Systems Account}

As was reviewed in the introduction, according to a behavior systems view (Timberlake, 1994, 2001; Timberlake \& Lucas, 1989; Timberlake \& K. M. Silva, 1995), a foraging animal progresses through a sequence of search modes and related responses entrained by proximity to 
food. Immediately after receiving food, a rat enters a postfood search mode characterized by behavior near the feeder. During the middle of the IFI, it enters general search, characterized by locomotion around the experimental chamber. As the time to food delivery approaches, it enters focal search, characterized by behavior focused on the feeder.

When provided with predictive stimuli signaling the presentation of food, search modes and related responses should become controlled by these stimuli. The extent to which a stimulus controls a particular search mode and related responses depends on its temporal and physical proximity to food. A stimulus immediately preceding food should evoke nosing in the feeder (a focal search response); stimuli presented in the middle of the IFI should evoke locomotion (a general search response); and a stimulus presented immediately following food should evoke behavior around the food site (a postfood search response).

Data from the present study's CRF groups showed that an interfood clock composed of four equal-length stimuli generates a sequence of responding consistent with the behavior systems framework - a replication of the results from other interfood clock studies with rats (K. M. Silva \& Timberlake, 1998b). During CRF, the greatest effect of the clock stimuli was to increase nosing in the feeder during the last two clock segments (S3 and especially S4), relative to a more modest increase in the noclock group, indicating that stimuli during the last half of the trial exert the most control over focal search (Palya \& Pevey, 1987).

The present experiment extended previous work by addressing the effects of PRF on the organization of interfood responding with and without a clock. The behavior system view suggests that PRF (relative to CRF) should affect the strength of general search, relative to focal search, and their expression during an interfood clock. Guided by the assumption that the reduction in the reliability of reward under PRF has an effect similar to that of lengthening the IFI, we expected CRF to produce relatively greater amounts of focal search behavior than PRF would and PRF to produce relatively greater amounts of general search behavior than CRF would (Timberlake $\&$ Lucas, 1989). Less focal search (nosing in the feeder) during S3 and S4 should occur, and the peak of general search (locomotion) should move closer to $\mathrm{S} 4$, because in a PRF schedule, that segment is, on average, closer to the middle of the actual IFI (time between food presentations) than in a CRF schedule and there is less competition from focal search behavior.

Four results from the present study support the assumption that PRF produces a perceived average greater distance between a clock segment and reward, relative to the CRF condition. First, the CRF-clock group nosed in the feeder during the last half of the trial segments more than the PRF-clock group did, as would be expected if the animals found the last two clock segments further away from the reward, on average, in the PRF conditions.
Second, the peak of absolute locomotion during the middle segments for the PRF-clock group was higher than the CRF-clock group's peak. Third, the ratio of general search in each clock segment to the maximum focal search (measured in the S4 segment) was higher across the first three clock segments for the PRF-clock group than for the CRF-clock group, showing greater general search under PRF. Fourth, the PRF- clock group's peak locomotion (during S3) was closer to the end of the clock than was the CRF-clock group's peak (during S2), again supporting the assumption that each clock segment was perceived as further from the reward under PRF. Further support for this last result is provided by K. M. Silva and Timberlake's (1998b) data showing that rats will shift their peak of locomotion toward the end of a clock in proportion to the increase in the distance from a reward. In that study, locomotion peaked in the second quarter of the IFI during clocks ranging between 12 and $198 \mathrm{sec}$.

Within a behavior systems view, work is needed on the acquisition of focal and general search and on tests to measure search states in more specific ways. For example, it appears that general and focal search overlap in expression during specific periods of the IFI. The presence of these states could be tested in more specific ways with summation or retardation tests (e.g., F. J. Silva, Timberlake, \& Gont, 1998), the presentation of moving probe stimuli (K. M. Silva \& Timberlake, 1997; Timberlake et al., 1982; Timberlake \& Washburne, 1989), or acoustic startle (Davis, 1989).

Both nosing-in-the-feeder and far-platform locomotion measures could probably be improved. Although the proportion of time spent on far-platform locomotion resembled total activity in the chamber (K. M. Silva, 1996; K. M. Silva \& Timberlake, 1998b), it actually referred only to behavior along the back wall. Nosing in the feeder was a robust and easily obtained measure of focal search, but it failed to capture the behavior of the PRF-no-clock group of attending to the feeder area without nosing. Finally, nosing in the feeder did not distinguish between continuous focused commitment to the feeder and the regular brief checking that occurs in earlier trial segments. These response forms could have been either slightly different responses or the same response, the duration of which was determined by competition with other behaviors.

\section{Factors Affecting the Emergence of Multiple Search Responses}

On the basis of how asymptotic peaks of nosing in the feeder and locomotion on the far platform developed over training, we can propose a general distinction between how general and focal search behaviors are organized over time and are related to clock stimuli. The clock groups' peaks of nosing in the feeder (focal search) during S3 and S4 developed as increases across sessions. In contrast, the peaks of locomotion (general search) during S2 (CRF-clock) and S3 (PRF-clock) emerged through maintenance of responding. Initially, locomotion was 
high in all the clock segments for both clock groups. At the end of training, though, it was high only in $\mathrm{S} 2$ for the $\mathrm{CRF}$-clock group and S3 for the PRF-clock group (see the right column of graphs in Figure 3). This suggests that initial locomotion is a response to clock stimuli and food presentation but that the maintenance of locomotion in $\mathrm{S} 3$ or S2 in the clock groups represents a form of acquisition that prevents decrease. Finally, the role of the clock cues in maintaining the clock groups' elevated locomotion in the middle of the IFI is consistent with research showing that rats' behavior occurring away from a feeder (e.g., leverpressing) during partially reinforced single-CS Pavlovian conditioning is higher in the presence of a CS than in its absence (Dudley \& Papini, 1997).

Response competition and dishabituation. When multiple behaviors occur in an IFI, response competition is often a plausible explanation for changes that occur across sessions. In the present data, one could argue that the clock stimuli control focal search and that locomotor responses fill in the remaining time. However, there were cases in which changes in one type of response were not accompanied by changes in the other. For example, locomotion during S1 decreased across training for the CRF groups, but nosing in the feeder showed no change. Similarly, for the PRF-clock group, locomotion decreased during S1, S2, and S4 without any change in nosing. Alternatively, dishabituation or slower habituation of reactions to clock stimuli in the PRF-clock group might have occurred because the clock components had unpredictable reward consequences (see Dudley \& Papini, 1997; Papini \& Dudley, 1997). However, it is not clear from this argument why S3 should be differentially affected by such dishabituation.

Density and frequency of reward. We might attribute elevated locomotion during S3 in the PRF relative to the CRF groups to reduced reward density and more unrewarded clock repetitions. This explanation could be addressed empirically by a CRF group that received 16 sessions of conditioning with a 96-sec IFI (with or without a clock). The reward density would be the same as that for the present PRF group, and each repetition of the clock sequence would end in reward, allowing us to infer the contribution of one of these variables. Results for exactly this condition were published in K. M. Silva and Timberlake's (1998b) study examining the effects of an interfood clock and IFI length on locomotion and nosing (appropriately, it was run at the same time as the present experiment). If reward density were the key to the differences in asymptotic responding between the present 48-sec CRF-clock and PRF-clock groups, we would expect the 96-sec CRF-clock group in K. M. Silva and Timberlake (1998b) to resemble the 48-sec PRF-clock group in terms of far-platform locomotion and nosing in the feeder. Instead, the 96-sec CRF-clock rats showed a peak of locomotion during S2 similar to that of the present 48-sec CRF-clock group, and not the peak in S3 shown by the present 48-sec PRF-clock group. Reward density, however, could account for the lower level of nosing at asymptote in both the 48 -sec PRF-clock group and the 96-sec CRF group.

A third factor possibly affecting the difference between the CRF-clock and the PRF-clock groups was the number of reinforcements received. As was mentioned, our primary goal was to equate the total number of trials received by all groups at 320 trials; this means, though, that for the PRF groups, only 160 of them were rewarded. All the groups showed stable responding after 200 trials, a result obtained in previous and current interfood clock studies (K. M. Silva \& Timberlake, 1998a, 1998b, 1999). This makes it seem unlikely that number of rewards is the key to the differences; however, as was noted in the Method section, we tested this more precisely by running both PRF groups for an additional 16 sessions in order to equate the total number of rewarded trials in the PRF and CRF groups. These data were not shown, because there were no changes in the measures for these groups across the additional sessions. For these reasons, we conclude that the PRF effects are due to reward unpredictability, rather than to reward density or number of rewards.

\section{Relevance to Timing Accounts}

Some of the present results potentially relate to other accounts of learning and behavior. It seems that scalar expectancy theory (SET; Gibbon \& Balsam, 1981) may relate to the present study, but it was not designed for multielement clocks, PRF, or multiple responses, and its focus has been on acquisition, rather than on asymptotic performance. Other things being equal, we would expect faster acquisition and greater responding in $\mathrm{S} 4$ in the PRF groups, because of the larger ratio of the effective IFI to S4 (Gibbon, Farrell, Locurto, Duncan, \& Terrace, 1980). However, our data do not support this prediction.

A better procedural fit than SET is Palya's (1993) bipolar model of interfood clock performance. This model specifies that stimuli correlated with successive portions of the latter half of the IFI are increasingly positive, whereas stimuli in successively earlier portions of the IFI are increasingly negative or aversive and stimuli in the middle of the IFI are relatively neutral. Changes in behavior are expected during stimuli correlated with these various portions of the IFI. Previous studies in which only a terminal response has been measured have found support for this model by showing that pigeons peck a key to produce the positive stimuli correlated with the last half of the IFI and peck a key that removes stimuli correlated with the first half of the IFI (Dinsmoor et al., 1986; Palya, 1993). Although the model does not explicitly predict control of multiple responses, the present results provide some support for it. Nosing in the feeder, which could be considered a terminal response, began to increase in S3, halfway through the IFI.

\section{Stimulus Control and Behavior Systems}

The present study adds evidence for the stimulus control of motivational modes and related responses (e.g., Burns \& Domjan, 1996; F. J. Silva, Timberlake, \& Cevik, 
1998; F. J. Silva, Timberlake, \& Gont, 1998; F. J. Silva et al., 1996; K. M. Silva \& Timberlake, 1998a, 1998b). We showed that the terminal response of nosing in the feeder is facilitated by interfood clock stimuli during the last half of a trial (S3 and S4), relative to a group without a clock (K. M. Silva \& Timberlake, 1998a, 1998b, 1999). This is consistent with previous experiments showing that terminal responses are facilitated by stimuli presented during the last half of the IFI, but not the first half (Gibbon \& Balsam, 1981; Palya, 1985; Palya \& Bevins, 1990; K. M. Silva \& Timberlake, 1998b).

The present experiment also adds to previous interfood clock studies (e.g., Dinsmoor et al., 1992) by showing that the effects of clock cues on nonterminal responding (e.g., locomotion) depend on the probability of reinforcement. Because locomotion typically peaks during the second segment of an IFI when the clock is present or absent during CRF (K. M. Silva \& Timberlake, 1998b), it has been suggested that locomotion occurring in the middle of an IFI may be controlled primarily by the passage of time (K. M. Silva \& Timberlake, 1998b) or contextual cues (e.g., Akins, Domjan, \& Gutierrez, 1994; Domjan, 1994; Matthews \& Lerer, 1987; K. M. Silva \& Timberlake, 1997), rather than by discrete predictive cues. However, the differential peaks in locomotion for the clock groups in the present study suggest that the probability of reinforcement can alter the pattern of locomotion across clock stimuli.

This result adds to K. M. Silva and Timberlake's (1999) data showing that the pattern of locomotion across clock stimuli can be altered. In that study, rats exposed to a sequence of clock stimuli that became increasingly longer (e.g., 4-8-12-24) showed a peak in locomotion during the second quarter of the IFI, as compared with a relatively flat pattern for rats receiving an increasingly shorter (e.g., 24-12-8-4) clock sequence. Although changing the characteristics of individual clock stimuli (i.e., duration) or the reward probability affects the pattern of locomotion across an IFI, this response still appears to be less tightly controlled by individual clock stimuli, as compared with nosing in the feeder (K. M. Silva \& Timberlake, 1999).

\section{Conclusions}

In the present study, we investigated how a Pavlovian PRF schedule affects multiple responses during a foursegment interfood clock. The pattern of responding across clock segments revealed that locomotion (general search) peaked during the middle of clock stimuli and nosing in the feeder (focal search) peaked during the final clock stimuli. In terms of amount of responding, PRF produced less nosing and relatively more locomotion than did CRF. These results support the behavior systems' view that a sequence of motivational modes and related responses are entrained by proximity to regular USs. Further work with different lengths of trial intervals and other general and focal search responses is needed to establish the generality of these results.

\section{REFERENCES}

Akins, C. A., Domjan, M., \& Gutierrez, G. (1994). Topography of sexually conditioned behavior in male Japanese quail (Coturnix japonica) depends on the CS-US interval. Journal of Experimental Psychology: Animal Behavior Processes, 20, 199-209.

BARNETT, S. A. (1975). The rat: A study in behavior. Chicago: University of Chicago Press.

BoaKes, R. A. (1977). Performance on learning to associate a stimulus with positive reinforcement. In H. Davis \& H. M. B. Hurwitz (Eds.), Operant-Pavlovian interactions (pp. 67-101). Hillsdale, NJ: Erlbaum.

BOAKES, R. A. (1979). Interactions between type I and type II processes involving positive reinforcement. In A. Dickinson \& R. A. Boakes (Eds.), Mechanisms of learning and motivation: A memorial volume to Jerzy Konorski (pp. 233-268). Hillsdale, NJ: Erlbaum.

Burns, M., \& Domjan, M. (1996). Sign tracking versus goal tracking in the sexual conditioning of male Japanese quail (Coturnix japonica). Journal of Experimental Psychology: Animal Behavior Processes, 22, 297-306

DaVEY, G. C. L., \& CLeland, G. G. (1982). Topography of signalcentered behavior in the rat: Effects of deprivation state and reinforcer type. Journal of the Experimental Analysis of Behavior, $\mathbf{3 8}$ 291-304.

DaVey, G. C. L., OAKley, D., \& Cleland, G. G. (1981). Autoshaping in the rat: Effects of omission on the form of the response. Journal of the Experimental Analysis of Behavior, 36, 75-91.

DAVIS, M. (1989). Sensitization of the acoustic startle reflex by footshock. Behavioral Neuroscience, 103, 495-503.

Dinsmoor, J. A. (1994). A comparison between the block clock and standard autoshaping procedures. Learning \& Motivation, 25, 313337.

Dinsmoor, J. A., Dougan, J. D., Pfister, J., \& Thiels, E. (1992). The autoshaping procedure as a residual block clock. Journal of the Experimental Analysis of Behavior, 58, 265-276.

DiNSMOOR, J. A., LEE, D. M., \& BRoWN, M. M. (1986). Escape from serial stimuli leading to food. Journal of the Experimental Analysis of Behavior, 46, 259-279.

DomJAN, M. (1994). Formulation of a behavior system for sexual conditioning. Psychonomic Bulletin \& Review, 1, 421-428.

Dudley, R. T., \& Papini, M. R. (1997). Amsel's frustration effect: A Pavlovian replication with control for frequency and distribution of rewards. Physiology \& Behavior, 61, 627-629.

EWER, R. F. (1971). The biology and behavior of a free-living population of black rats (Rattus rattus). Animal Behavior Monographs, 4 127-174.

FANSELOW, M. S. (1994). Neural organization of the defensive behavior system responsible for fear. Psychonomic Bulletin \& Review, $\mathbf{1}_{2}$ 429-438.

FERSTER, C. B., \& SKINNER, B. F. (1957). Schedules of reinforcement. New York: Appleton-Century-Crofts.

Gibbon, J., \& Balsam, P. (1981). Spreading association in time. In C. M. Locurto, H. S. Terrace, \& J. Gibbon (Eds.), Autoshaping and conditioning theory (pp. 218-253). New York: Academic Press.

Gibbon, J., Farrell, L., Locurto, C. M., Duncan, H. J., \& Terrace, H. S. (1980). Partial reinforcement in autoshaping with pigeons. $A n-$ imal Learning \& Behavior, 8, 45-59.

GibBs, C. M., Latham, S. B., \& Gormezano, I. (1978). Classical conditioning of the rabbit nictitating membrane response: Effect of reinforcement schedule on response maintenance and resistance to extinction. Animal Learning \& Behavior, 6, 209-215.

Holland, P. C. (1980). CS-US interval as a determinant of the form of Pavlovian appetitive conditioned responses. Journal of Experimental Psychology: Animal Behavior Processes, 6, 155-174.

InNIS, N. K., SimMELHAG-GRANT, V. L., \& STADDON, J. E. R. (1983). Behavior induced by periodic food delivery: The effects of interfood interval. Journal of the Experimental Analysis of Behavior, 39, 309-322.

Jenkins, H. M., Barrera, F. J., Ireland, C., \& Woodside, B. (1978). Signal-centered action patterns of dogs in appetitive classical conditioning. Learning \& Motivation, 9, 272-296.

KONORSKI, L. (1967). Integrative activity of the brain. Chicago: University of Chicago Press. 
LAWler, C. P., \& Cohen, P. S. (1992). Temporal patterns of scheduleinduced drinking and pawgrooming in rats exposed to periodic food. Animal Learning \& Behavior, 20, 266-280.

Lucas, G. A., Timberlake, W., \& Gawley, D. J. (1988). Adjunctive behavior of the rat under periodic food delivery in a 24-hour environment. Animal Learning \& Behavior, 16, 19-30.

Macintosh, N. J. (1974). The psychology of animal learning. London: Academic Press.

Matthews, T. J., Bordi, F., \& Depollo, D. (1990). Schedule-induced kinesic and taxic behavioral stereotypy in the pigeon. Journal of Experimental Psychology: Animal Behavior Processes, 16, 335-344.

MATTHEWs, T. J., \& LERER, B. E. (1987). Behavior patterns in pigeons during autoshaping with an incremental conditioned stimulus. Animal Learning \& Behavior, 15, 69-75.

PALYA, W. L. (1985). Sign-tracking with an interfood clock. Journal of the Experimental Analysis of Behavior, 43, 321-330.

PALYA, W. L. (1993). Bipolar control in fixed interfood intervals. Journal of the Experimental Analysis of Behavior, 60, 354-359.

Palya, W. L., \& Bevins, R. A. (1990). Serial conditioning as a function of stimulus, response, and temporal dependencies. Journal of the Experimental Analysis of Behavior, 53, 65-85.

Palya, W. L., \& Bowers, M. T. (2003). Stimulus control in fixed interfood intervals. Animal Learning \& Behavior, 31, 22-34.

Palya, W. L., \& Pevey, M. E. (1987). Serial conditioning as a function of parametric variations of an interfood clock. Animal Learning \& Behavior, 15, 249-262.

PAPINI, M. R., \& DUDLEY, R. T. (1997). Consequences of surprising reward omissions. Review of General Psychology, 1, 175-197.

Pavlov, I. P. (1927). Conditioned reflexes (G. V. Anrep, Trans.). London: Oxford University Press.

Pecoraro, N. C., Timberlake, W. D., \& Tinsley, M. (1999). Incentive downshifts evoke search repertoires in rats. Journal of Experimental Psychology: Animal Behavior Processes, 25, 153-167.

REID, A. K., BACHA, G., \& Moran, C. (1993). The temporal organization of behavior on periodic food schedules. Journal of the Experimental Analysis of Behavior, 59, 1-27.

Silva, F. J., Silva, K. M., \& PEAR, J. J. (1992). Sign- versus goal-tracking: Effects of conditioned-stimulus-to-unconditioned-stimulus distance. Journal of the Experimental Analysis of Behavior, 57, 17-31.

Silva, F. J., \& Timberlake, W. (2000). A clarification on the nature of backward excitatory conditioning. Learning \& Motivation, 31, 67-80

Silva, F. J., Timberlake, W., \& CeviK, M. O. (1998). A behavior systems approach to the expression of backward associations. Learning \& Motivation, 29, 1-22.

SiLva, F. J., TimberLAKE, W., \& Gont, R. S. (1998). Spatiotemporal characteristics of serial CSs and their relation to search modes and response form. Animal Learning \& Behavior, 26, 299-312.

Silva, F. J., Timberlake, W., \& Koehler, T. L. (1996). A behavior systems approach to bidirectional excitatory conditioning. Learning \& Motivation, 27, 130-150.

SILVA, K. M. (1996). Appetitive behavior during an interfood clock. Unpublished doctoral dissertation, Indiana University, Bloomington.

Silva, K. M., \& Timberlake, W. (1997). A behavior systems view of conditioned states during long and short CS-US intervals. Learning \& Motivation, 28, 465-490.
Silva, K. M., \& Timberlake, W. (1998a). A behavior systems view of responding to probe stimuli during an interfood clock. Animal Learning \& Behavior, 26, 313-325.

SiLVA, K. M., \& TiMBERLAKE, W. (1998b). Organization and temporal properties of appetitive behavior in rats. Animal Learning \& Behavior, 26, 182-195.

SiLVA, K. M., \& TimberlaKe, W. (1999). Rats' behavior during an interfood clock is altered by the temporal pattern of interfood stimuli. Learning \& Motivation, 30, 183-200.

STADDON, J. E. R. (1977). Schedule-induced behavior. In W. K. Honig \& J. E. R. Staddon (Eds.), Handbook of operant behavior (pp. 125152). New York: Prentice-Hall.

Staddon, J. E. R., \& Simmelhag, V. L. (1971). The "superstition" experiment: A re-examination of its implications for the principles of adaptive behavior. Psychological Review, 78, 3-43.

Steiniger, F. (1950). Beiträge zur Soziologie und sonstigen Biologie der Wanderratte. Zeitschrift für Angewandte Zoologie, 7, 356-379.

Telle, H. J. (1966). Beitrag zur Kenntnis der Verhaltensweise von Ratten, vergleichend dargestellt bei Rattus norvegicus und Rattus rattus. Zeitschrift für Angewandte Zoologie, 53, 129-196.

Timberlake, W. (1983). The functional organization of appetitive behavior: Behavior systems and learning. In M. D. Zeiler \& P. Harzem (Eds.), Advances in analysis of behaviour: Vol. 3. Biological factors in learning (pp. 177-221). Chichester, U.K.: Wiley.

TimberlaKe, W. (1994). Behavior systems, associationism, and Pavlovian conditioning. Psychonomic Bulletin \& Review, 1, 405-420.

Timberlake, W. (2001). Motivational modes in behavior systems. In R. R. Mowrer \& S. B. Klein (Eds.), Handbook of contemporary learning theories (pp. 155-209). Hillsdale, NJ: Erlbaum.

Timberlake, W., \& Lucas, G. (1985). The basis of superstitious behavior: Chance contingency, stimulus substitution, or appetitive behavior. Journal of the Experimental Analysis of Behavior, 44, 279299.

Timberlake, W., \& Lucas, G. (1989). Behavior systems and learning: From misbehavior to general principles. In S. B. Klein \& R. R. Mower (Eds.), Contemporary learning theories: Instrumental conditioning theory and the impact on biological constraints on learning (pp. 237275). Hillsdale, NJ: Erlbaum.

Timberlake, W., \& Silva, F. J. (1994). Observation of behavior, inference of function, and the study of learning. Psychonomic Bulletin \& Review, 1, 73-88.

Timberlake, W., \& Silva, K. M. (1995). Appetitive behavior in psychology, ethology, and behavior systems. In N. Thompson (Ed.), Perspectives in ethology: Vol. 11. Behavioral design (pp. 212-254). New York: Plenum.

Timberlake, W., Wahl, G., \& KIng, D. (1982). Stimulus and response contingencies in the misbehavior of rats. Journal of Experimental Psychology: Animal Behavior Processes, 8, 62-85.

Timberlake, W., \& Washburne, D. L. (1989). Feeding ecology and laboratory predatory behavior toward live and artificial moving prey in seven rodent species. Animal Learning \& Behavior, 17, 1-10.

(Manuscript received May 18, 2004; revision accepted for publication September 7, 2004.) 